\title{
Development of Static Magnetic Detection Anti-Collision System While Drilling
}

\author{
Binbin Diao' ${ }^{1}$, Deli Gao ${ }^{1}$, Genkui Li \\ ${ }^{1}$ China University of Petroleum, Beijing, China; \\ ${ }^{2}$ Sinopec Jiangsu Oil Engineering Co.,Ltd, Jiangsu, China.
}

\begin{abstract}
The technique of adjacent well anti-collision is one of key technologies in the cluster well directional drilling project. Based on research of the magnetic field distribution around casing string, a method is proposed to determine the distance between adjacent wells by measuring the magnetic field generated by the casings while drilling at two different measurement depths of existing well. A prototype of the static magnetic detection anti-collision system while drilling is developed, which comprises the underground probe, the driver sub, the interface box and the software for calculating the relative position between adjacent wells. The prototype can be used with the traditional MWD (Measurement While Drilling) tool on the site. The ground modeling experiment indicates that the distance between adjacent wells calculated by the prototype can properly accord with the results of theoretical analysis and meet the actual requirements of the cluster well drilling engineering. Such the system is expected to provide a highly efficient means for anti-collision in cluster wells.
\end{abstract}

Keywords: Cluster Wells; Directional Drilling; Adjacent Well Distance; Anti-Collision; Magnetic Guidance.

\section{Introduction}

In order to bridge the gap between the supply and demand of oil and gas in China, it is highly necessary to develop the unconventional, low-permeability and marine oil and gas resources. Meanwhile, the potential of the old and mature oil filed should also be further exploited. The more demands for the cluster well projects are put forward in efficient development of such oil and gas resources. In recent years, the cases of collision between vertical and build-up sections have taken place more frequently in cluster wells and adjustment wells, which often need repetitive repair work. Once the existing drilling has been drilled through, it will cause serious economic losses.

The traditional anti-collision method is achieved by adjacent well distance scanning and separation factor calculation so as to judge the collision probability of adjacent wells [1,2]. Benny Poedjono and his colleagues $[3,4]$ proposed the concept of oriented separation factor and the calculating method of oriented separation factor. However, these methods cannot prevent the accumulated error in well trajectory calculation and thus fail to meet the actual needs of cluster well drilling engineering. In recent decades, thanks to the invention of magnetic guidance tools, the anti-collision technology has witnessed leapfrog development. These tools can precisely measure the distance and direction from one well to another. At present, such magnetic guidance tools includes SWG (Single Wire Guidance Tool), RMRS (Rotating Magnet Ranging System) and MagTraC. When SWG and RMRS are used, the probe should be put in the well being drilled and a signal source should be put in the existing well [5-8]. MagTraC combines the high-precision SuperEye or GoldenEye probe and the traditional MWD. It measures the magnetic field generated by the casings of existing wells, then the distance and direction from the drilling bit to the existing well can be calculated based on the magnetic signals $[9,10]$. Currently, the tool has been successfully applied in the anti-collision projects of over 300 cluster wells. Besides, the tool has been successfully used in in SAGD horizontal wells and relief wells.

The research of active anti-collision technology is still relatively backward in China and there is not a type of commercialized anti-collision tool yet. In order to lower the risks of collision, reduce the drilling cost and enhance the ability of directional drilling service, there is an urgent need to research and develop active anti-collision technology. 


\section{Working principle of static magnetic detection anti-collision system while drilling}

As shown in Fig.1, the improve probe is the key hardware of the static magnetic detection anti-collision system while drilling. First, the information of the well trajectory design and the adjacent well's actual trajectory is obtained. Second, the closest distance and the separation factor from the well being drilled to adjacent wells are calculated to determine the well sections that need anti-collision. Then the improved probe (as shown in Fig.2) is installed in the bottom hole assembly where is the position of traditional MWD probe. When the drilling bit touches the section that needs anti-collision, the data (including the tri-axial gravity acceleration $G_{x}, G_{y}$ and $G_{z}$ incurred in the gravity field and two set of tri-axial magnetic induction $B_{1 x}, B_{1 y}, B_{1 z}, B_{2 x}, B_{2 y}$ and $B_{2 z}$ incurred by the casings and geomagnetic filed) detected by the improved probe is transmitted to the ground computer through the MWD wireless data transmission technology. The analysis software installed in the computer can calculate the relative position between the improve probe and the casings based on the gathered data. Then, the relative position of the drilling bit and the existing well can be determined. These results provide the scientific basis for controlling the trajectory of the well being drilled to avoid collision with adjacent wells.

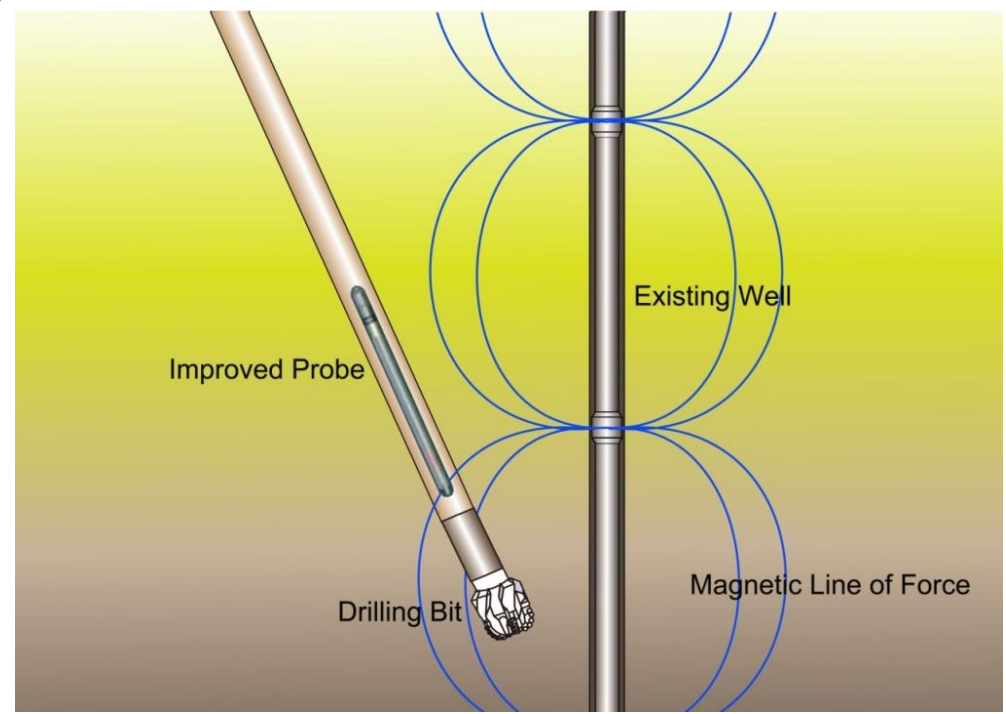

Fig. 1 Working principle of static magnetic detection anti-collision system while drilling

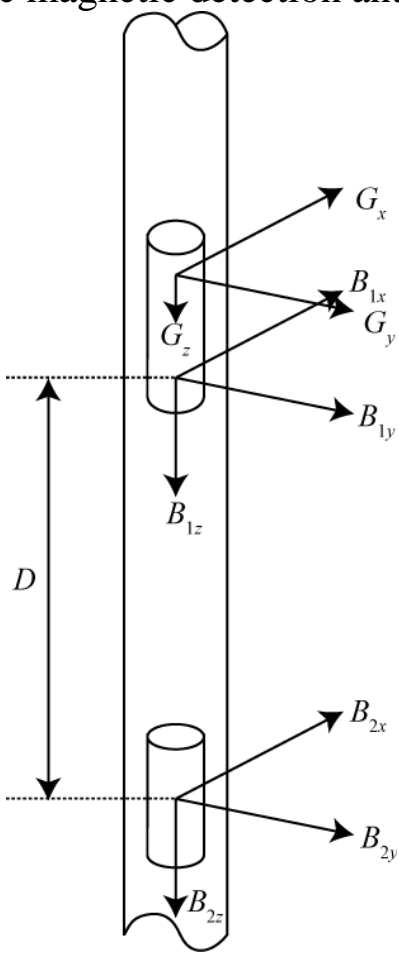

Fig. 2 Schematic diagram of the improved MWD probe 


\section{The magnetic distribution around casing string}

The magnetic field of the casing pipe can have many different sources, among which the main source is the magnetic field incurred in its mechanical processes and magnetic leakage flux. A casing can be regarded as a magnetic dipole. As shown in Fig.3, when several casings are combined in an existing well, several dipoles rank along the well. In this process, the north magnetic pole of one casing can be connected with the north or south magnetic pole of another casing.

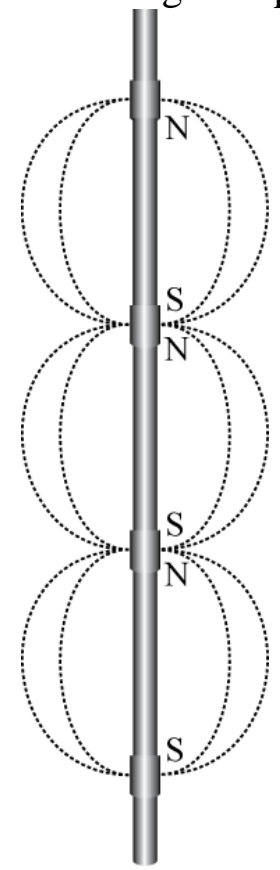

Fig. 3 The magnetic distribution around the casing string

Since the distance between the measuring point and the casing string would not surpass the length of a casing, the magnetic field distribution around the casing is similar to that around the dipoles. As shown in Fig.4, the magnetic field distribution around the casing can be described by:

$$
\begin{aligned}
& B_{z}=\frac{Q}{4 \pi}\left(\frac{z-l}{R_{1}^{3}}-\frac{z+l}{R_{2}^{3}}\right) \\
& B_{r}=\frac{Q r}{4 \pi}\left(\frac{1}{R_{1}^{3}}-\frac{1}{R_{2}^{3}}\right)
\end{aligned}
$$

where, $Q$ is the size of the magnetic charge; $B_{z}$ and $B_{r}$ are the component of the magnetic induction intensity along the $z$ axis and $r$ axis; $l$ is half the length of the casing pipe of the adjacent drilled well; $R_{1}$ is the distance from the negative magnetic charge to the measurement point; $R_{2}$ is the distance from the positive magnetic charge to the measurement point.

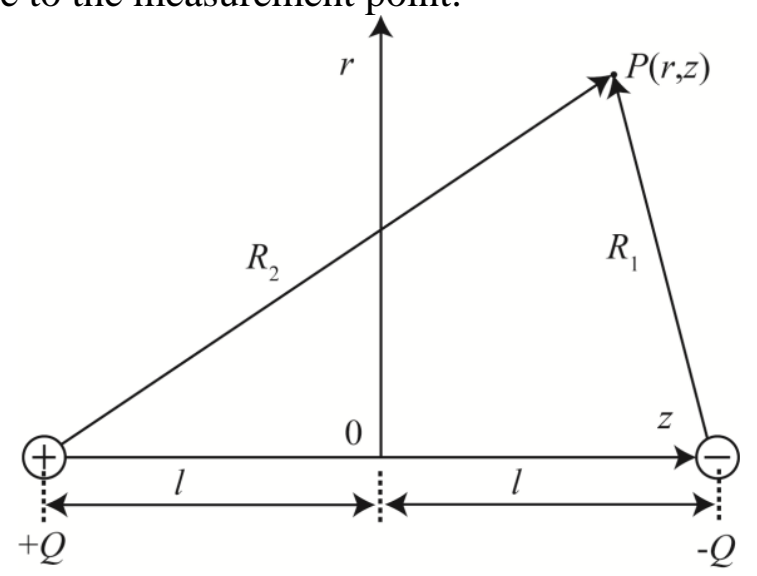

Fig.4 Calculation model for the magnetic field around the dipoles 


\section{Calculation of the relative position between two wells}

As shown in Fig.5 and Fig.6, the relative position between the improved probe and the casing string of the existing well can be determined by the radial spacing $r$ between the improved probe and the casing string and the angle $A_{h r}$ from the high side of the well being drilled and the unit vector $\hat{r}$.

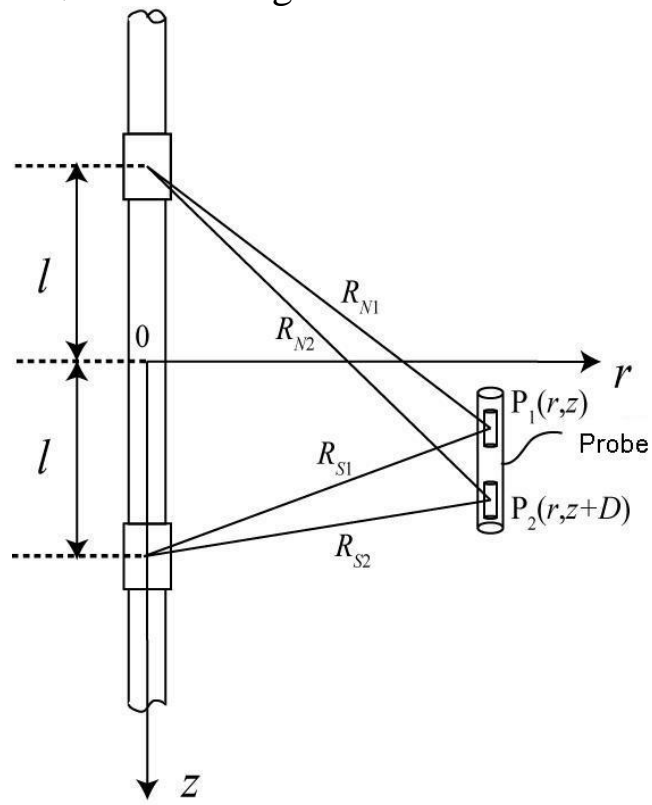

Fig. 5 Calculation model of the distance between the probe and the adjacent well

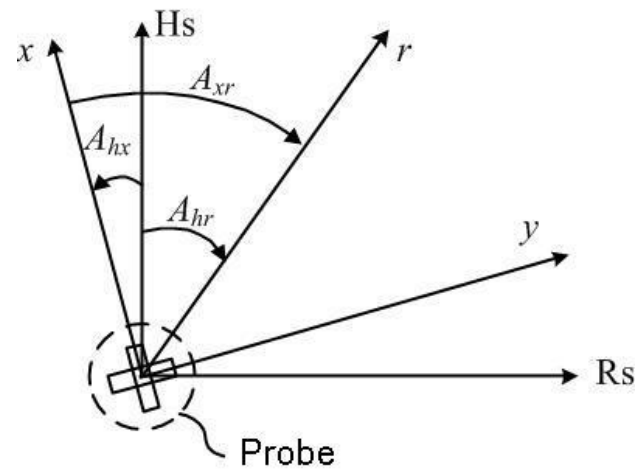

Fig. 6 Calculation model of the direction from the probe to the adjacent well

As shown in Fig.5, based on the magnetic field distribution rule around the casing string, the magnetic induction intensity detected by the two sensors in the improve probes can be described by:

$$
\begin{aligned}
& B_{1 z}=\frac{Q}{4 \pi}\left(\frac{z-l}{R_{N 1}^{3}}-\frac{z+l}{R_{S 1}^{3}}\right) \\
& B_{1 r}=\frac{Q r}{4 \pi}\left(\frac{1}{R_{N 1}^{3}}-\frac{1}{R_{S 1}^{3}}\right) \\
& B_{2 z}=\frac{Q}{4 \pi}\left(\frac{(z+D)-l}{R_{N 2}^{3}}-\frac{(z+D)+l}{R_{S 2}^{3}}\right) \\
& B_{2 r}=\frac{Q r}{4 \pi}\left(\frac{1}{R_{N 2}^{3}}-\frac{1}{R_{S 2}^{3}}\right)
\end{aligned}
$$

Where, $D$ is the distance between the high-precision inclinometer and the high-precision three-axis fluxgate sensor; $B_{1 z}$ and $B_{2 z}$ represent respectively the measured axial magnetic induction intensity by the two high-precision three-axis fluxgate sensors; $B_{1 r}$ and $B_{2 r}$ represent respectively the measured radial magnetic induction intensity by the two high-precision three-axis fluxgate sensors. $B_{1 r}$ and $B_{2 r}$ can be obtained by: 


$$
\begin{aligned}
& B_{1 r}=\sqrt{B_{1 x}^{2}+B_{1 y}^{2}} \\
& B_{2 r}=\sqrt{B_{2 x}^{2}+B_{2 y}^{2}}
\end{aligned}
$$

Where, $B_{1 x}$ and $B_{2 x}$ represent respectively the measured magnetic induction intensity component by the two $x$-axis fluxgate sensors; $B_{1 y}$ and $B_{2 y}$ represent respectively the measured magnetic induction intensity component by the two $y$-axis fluxgate sensors.

With Eqs. (3) and (5), the following is obtained:

$$
\begin{aligned}
& B_{1 z}=\frac{Q}{4 \pi}\left\{z\left(\frac{1}{R_{N 1}^{3}}-\frac{1}{R_{S 1}^{3}}\right)-l\left(\frac{1}{R_{N 1}^{3}}+\frac{1}{R_{S 1}^{3}}\right)\right\} \\
& B_{2 z}=\frac{Q}{4 \pi}\left\{(z+D)\left(\frac{1}{R_{N 2}^{3}}-\frac{1}{R_{S 2}^{3}}\right)-l\left(\frac{1}{R_{N 2}^{3}}+\frac{1}{R_{S 2}^{3}}\right)\right\}
\end{aligned}
$$

Because the size of magnetic poles $Q$ is not fixed value and often hard to obtain, the parameter $Q$ should be eliminated in the calculation. The ratio of the axial and radial direction components of the magnetic induction intensity can be described by:

$$
\begin{aligned}
& \frac{B_{1 z}}{B_{1 r}}=\frac{z}{r}-\frac{l}{r} \frac{\left(1+\left(\frac{z}{r}-\frac{l}{r}\right)^{2}\right)^{3 / 2}+\left(1+\left(\frac{z}{r}+\frac{l}{r}\right)^{2}\right)^{3 / 2}}{\left(1+\left(\frac{z}{r}-\frac{l}{r}\right)^{2}\right)^{3 / 2}-\left(1+\left(\frac{z}{r}+\frac{l}{r}\right)^{2}\right)^{3 / 2}} \\
& \frac{B_{2 z}}{B_{2 r}}=\frac{z+D}{r}-\frac{l}{r} \frac{\left(1+\left(\frac{z+D}{r}-\frac{l}{r}\right)^{2}\right)^{3 / 2}+\left(1+\left(\frac{z+D}{r}+\frac{l}{r}\right)^{2}\right)^{3 / 2}}{\left(1+\left(\frac{z+D}{r}-\frac{l}{r}\right)^{2}\right)^{3 / 2}-\left(1+\left(\frac{z+D}{r}+\frac{l}{r}\right)^{2}\right)^{3 / 2}}
\end{aligned}
$$

With Eqs. (11) and (12), the following is obtained:

$$
\begin{gathered}
\frac{z}{r}-\frac{l}{r} \frac{\left(1+\left(\frac{z}{r}-\frac{l}{r}\right)^{2}\right)^{3 / 2}+\left(1+\left(\frac{z}{r}+\frac{l}{r}\right)^{2}\right)^{3 / 2}}{\left(1+\left(\frac{z}{r}-\frac{l}{r}\right)^{2}\right)^{3 / 2}-\left(1+\left(\frac{z}{r}+\frac{l}{r}\right)^{2}\right)^{3 / 2}}-\frac{B_{1 z}}{B_{1 r}}=0 \\
\frac{z+D}{r}-\frac{l}{r} \frac{\left(1+\left(\frac{z+D}{r}-\frac{l}{r}\right)^{2}\right)^{3 / 2}+\left(1+\left(\frac{z+D}{r}+\frac{l}{r}\right)^{2}\right)^{3 / 2}}{\left(1+\left(\frac{z+D}{r}-\frac{l}{r}\right)^{2}\right)^{3 / 2}-\left(1+\left(\frac{z+D}{r}+\frac{l}{r}\right)^{2}\right)^{3 / 2}}-\frac{B_{2 z}}{B_{2 r}}=0
\end{gathered}
$$

Therefore, the radial spacing $r$ can be obtained by solving the following nonlinear equations. The initial value can be obtained based on the results of the closest distance scanning between the planned trajectory of the well being drilled and the actual drilling trajectory of the existing well.

As shown in Fig.6, the angle $A_{h r}$ can be described by:

$$
\begin{aligned}
& A_{h r}=A_{h x}+A_{x r} \\
& A_{h x}=\arctan \left(\frac{G_{x}}{G_{y}}\right)
\end{aligned}
$$




$$
A_{x r}=\arctan \left(\frac{B_{c x}}{B_{c y}}\right)
$$

Where, $G_{x}$ and $G_{y}$ represent respectively the gravity component detected by the $x$-axis and $y$-axis accelerometers; $B_{c x}$ and $B_{c y}$ represent respectively the $x$-axis and $y$-axis components of the magnetic field generated by casing string.

In application, $B_{c x}$ and $B_{c y}$ can be calculated by combining the data measured by the high-precision inclinometer and the magnetic induction intensity of the geomagnetic field.

$$
\begin{aligned}
& B_{c x}=B_{1 x}-B_{e x} \\
& B_{c y}=B_{1 y}-B_{e y} \\
& B_{e x}=B_{e}(\cos \Theta \sin A \cos \alpha+\cos \Theta \cos A \cos I \sin \alpha-\sin \Theta \sin I \sin \alpha) \\
& B_{e y}=B_{e}(\cos \Theta \cos A \cos I \cos \alpha+\sin \Theta \sin I \cos \alpha-\cos \Theta \sin A \sin \alpha)
\end{aligned}
$$

Where, $B_{e}$ is the magnetic induction intensity of the geomagnetic field; $I$ is the deviation angle; $A$ is the azimuth; $\alpha$ is the roll angle of the inclinometer; $\Theta$ is the earth's magnetic dip angle.

\section{Development of the hardware of static magnetic detection anti-collision system while drilling}

In order to measure the adjacent well distance while drilling, the improved probe of the system must be installed in the bottom hole assembly. And, make sure to the probe as close as possible to the drilling bit. However, in reality, behind the drilling bit is usually the downhole motor. It requires the mud-pulse to transmit the data detected by the probe to the ground. Therefore, the best position for the improved probe is behind the downhole motor, as shown in Fig7.

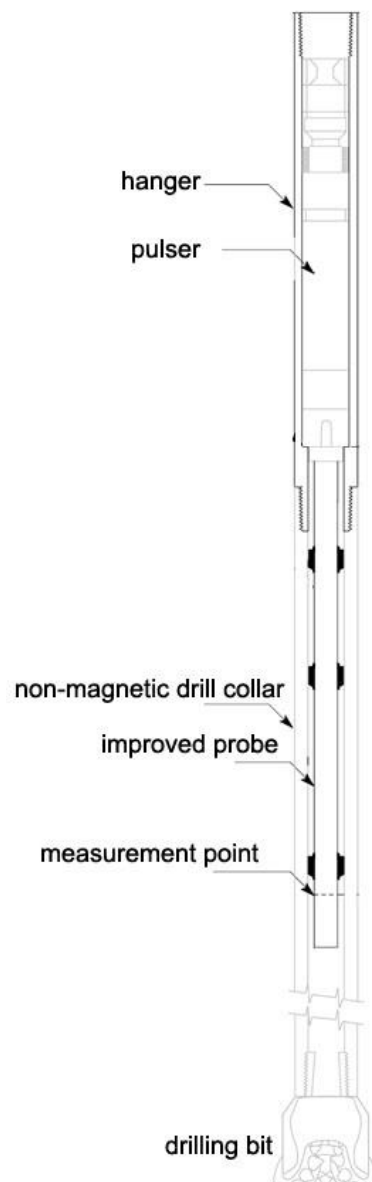

Fig. 7 Schematic diagram of bottom hole assembly 


\section{Downhole Subsystem}

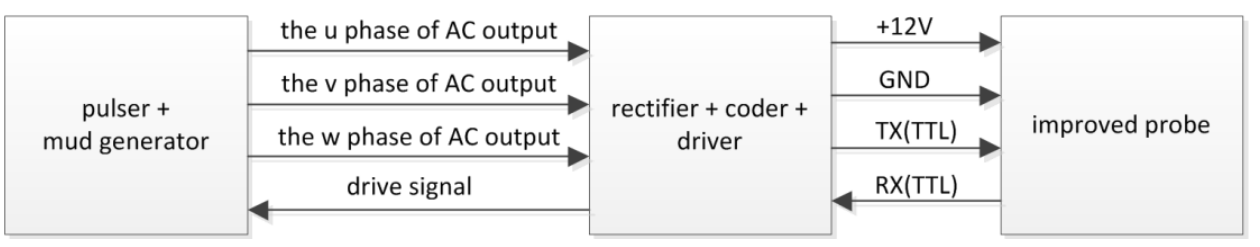

Fig. 8 Schematic diagram of the downhole subsystem

As shown in Fig.8, the downhole subsystem of the static magnetic detection anti-collision system while drilling mainly includes the pulser, the mud generator, the improved probe, and the sub with rectifier, coder and driver functionality (as shown in Fig.9).

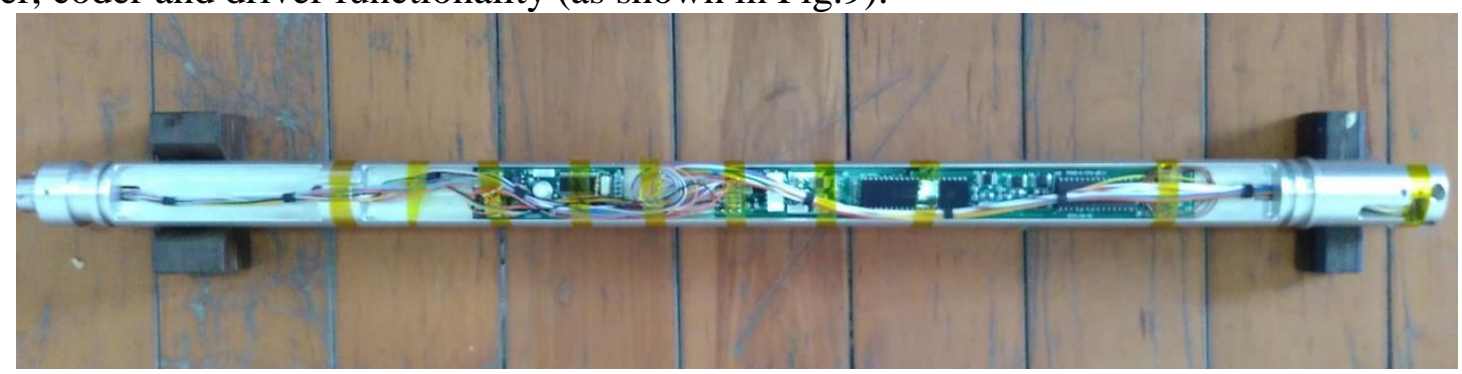

Fig. 9 The sub with rectifier, coder and driver functionality

The downhole subsystem can work under two models: MWD model or anti-collision model. The model can be switched by the code of the switch pump. Each set needs to upload 28 bytes of data in anti-collision model, which takes about 5 minutes.

The main function of the improved probe is to detect the geomagnetic field, the magnetic field generated by casing string, and gravity field. Then, the detected signals is processed and transmitted to the ground by the data transmission technology of MWD. As shown in Fig.10, the probe mainly includes the high-precision inclinometer, the high-precision tri-axis fluxgate sensors, and the data acquisition circuit.

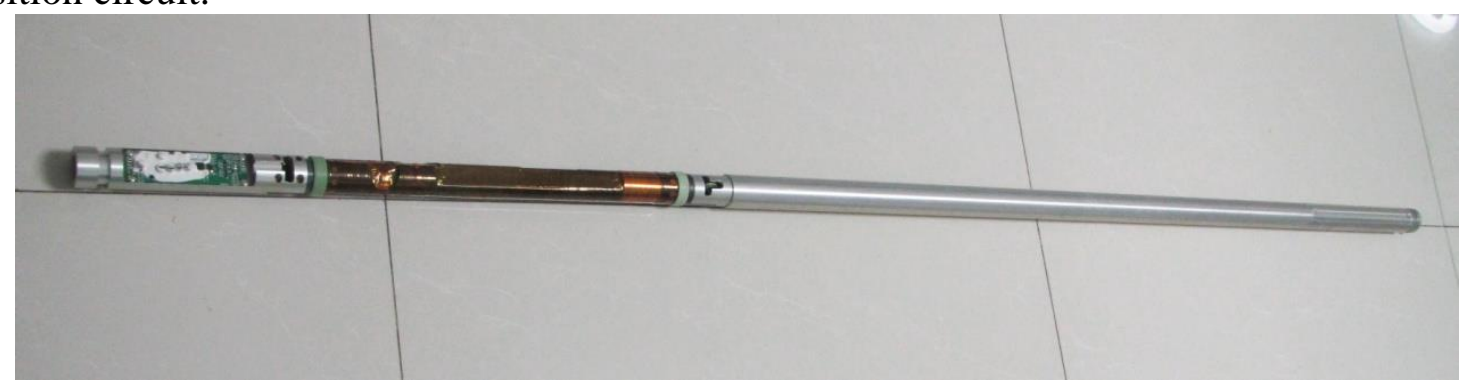

Fig. 10 The photo of the improved probe

\section{Simulation experiment and result analysis}

The experiment site is near the farming fields, with the closest highroad some 1000m away. The nearest high-voltage electric tower is about $800 \mathrm{~m}$ away; the nearest low-voltage electric wire is about $100 \mathrm{~m}$ away. Besides, there is no metal cable and pipeline under the ground. Generally speaking, the ambient magnetic field is relatively stable. To simulate the casing pipe of the adjacent well, three 5" casings are placed $0.35 \mathrm{~m}$ under the ground with a total length of $28.47 \mathrm{~m}$. In the experiment, the probe shown in Fig.10 is needed. Meanwhile, as shown in Fig.11, the interface box and computer are needed. The interface box is used to transmit the signal to the computer and to provide $48 \mathrm{~V}$ power for the probe. The relative position from the probe to the casing string can be obtained by the ranging software installed in the computer. 


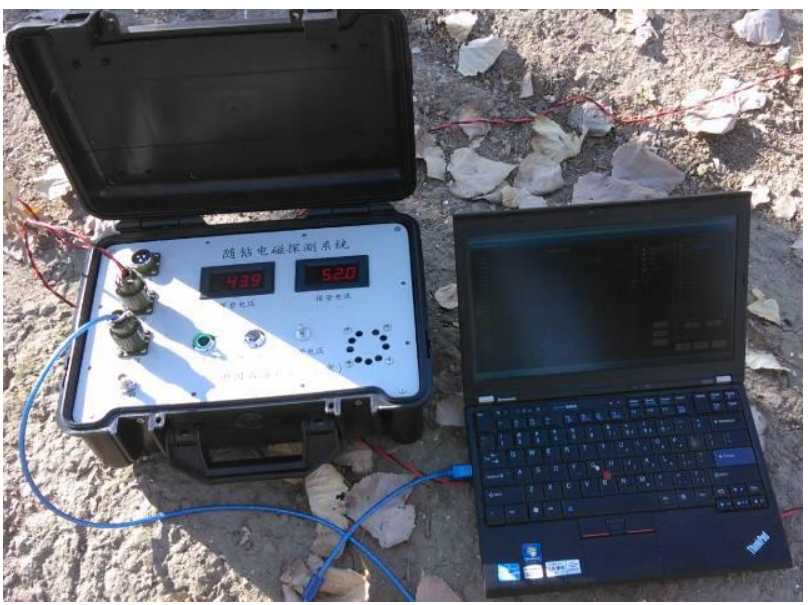

Fig. 11 Interface box and computer

When the probe is $4 \mathrm{~m}$ away from the casing string, translate the probe along the axial direction of the casing string, measure and save the data once every $0.5 \mathrm{~m}$. Each time, the measurement records about 10 seconds. The measured data is shown in Fig.12. In the drilling site, if the all data are transmitted to the ground, a lot of time would be wasted. Hence, the data recorded at each measuring point should also be extracted. The calculated value of the radial spacing between the probe and the casing string is $3.76 \mathrm{~m}$. So the calculation error is $6 \%$, which meets the requirement of anti-collision.
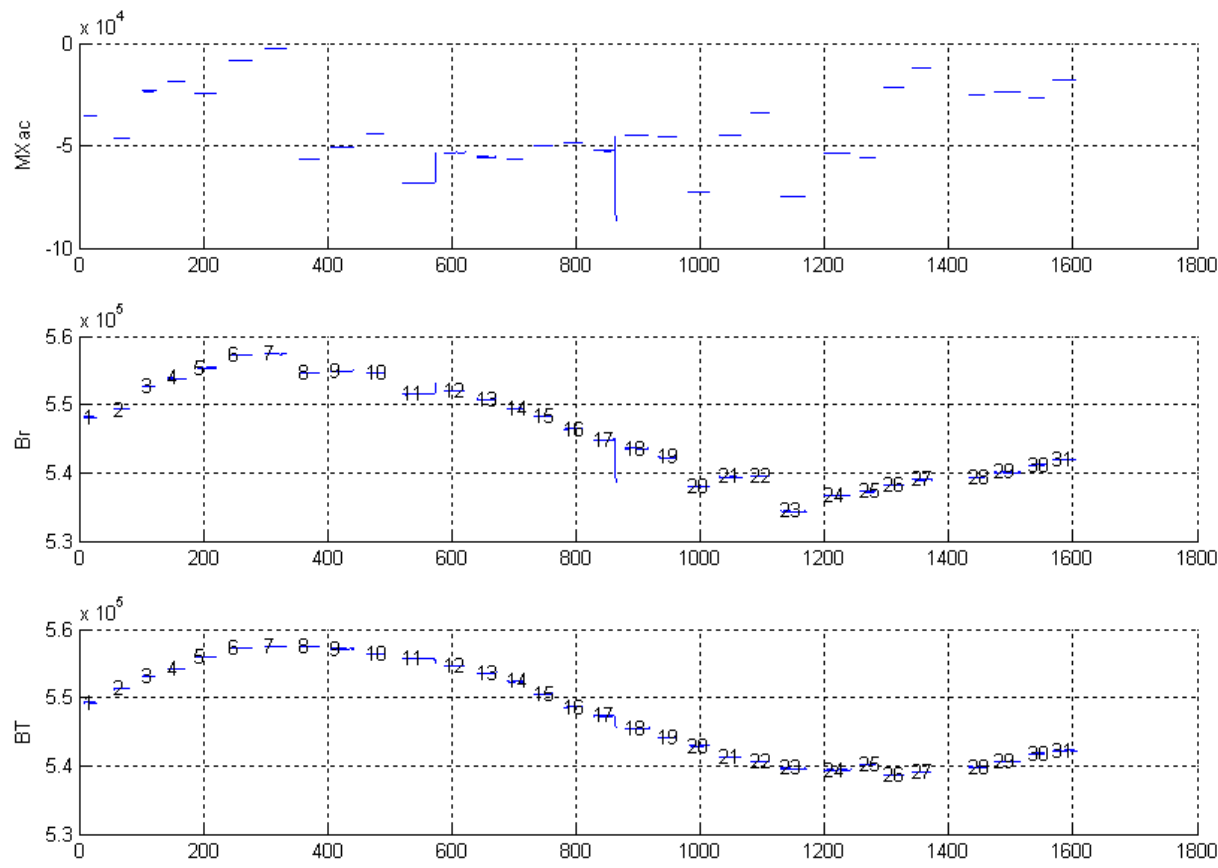

Fig. 12 Data recorded when the probe is $4 \mathrm{~m}$ away from the casing pipe

\section{Conclusion}

(1) In response to the urgent need for anti-collision technology in cluster wells and adjustment wells, a method of determining the adjacent well distance is proposed based on the magnetic field distribution rule around the casing string.

(2) A prototype of the static magnetic detection anti-collision system while drilling is developed. The prototype with the traditional MWD can be used on site.

(3) The simulation experiment indicates that the adjacent well distance calculated by the sample machine can properly accord with the results of theoretical analysis and meet the actual requirements of the cluster well drilling. 
(4) In drilling engineering, the adjacent well distance calculated by the system may be interfered by many factors such as stratum and ferromagnet. In the future, more simulation experiments and theoretical analysis are needed to increase the calculation precision of the adjacent well distance.

\section{Acknowledgements}

The authors gratefully acknowledge the financial support of the Natural Science Foundation of China (NSFC, 51221003, 51304221). This research was also supported by Science Foundation of China University of Petroleum, Beijing (2462014YJRC035).

\section{References}

[1] McNair G A, Lance S J, Codling J, et al. Implementation of a new risk based well collision avoidance method. SPE 92554, 2005.

[2] Zhang Xiaocheng, Liu Yajun, Wang Kunjian, et al. Application of path control technology of overall infill drilling on offshore cluster well group. Oil Forum, 2010, 5:13-17.

[3] Poedjono B, Akinniranye G, Conran G, et al. Minimizing the risk of well collisions in land and offshore drilling. SPE/IADC 108279, 2007.

[4] Poedjono B, Lombardo G J, Phillips W. Anti-collision risk management standard for well placement. SPE 121040, 2009.

[5] Mallary C R, Williamson H S, Pitzer R, et al. Collision avoidance using a single wire magnetic ranging technique at Milne point, Alaska. SPE 39389, 1998.

[6] Kuckes A F. Borehole guidance system having target wireline. US5074365, 1991.

[7] Kuckes A F. Rotating magnet for distance and direction measurements from a first borehole to a second borehole. US5589775, 1996.

[8] Nekut A G, Kuckes A F and Pitzer R G. "Rotating magnet ranging - a new drilling guidance technology" 8th one day conference on horizontal well technology. Canadian Sections SPE / Petroleum Society, November 7th, 2001.

[9] James N T. Method to detect deviations from a wellplan while drilling in the presence of magnetic interference. US6530154, 2003.

[10] Duncan L. MWD Ranging-A Hit and a Miss. Oil Gas-European Magazine, 2013, 39(1): 24-26. 